\title{
Assessment of Intracellular Calcium and Plasmalemmal Membrane Potential in Cryopreserved Metaphase II Mouse Oocytes
}

Omar Farhan Ammar ( $\sim$ Omar.ammar.iq@gmail.com )

https://orcid.org/0000-0001-8048-9747

Therishnee Moodley

University of Dundee School of Medicine

\section{Research note}

Keywords: Mouse Oocytes, IVF, Cryopreservation, KATP channels, Intracellular Calcium, Membrane potential

Posted Date: August 3rd, 2020

DOI: https://doi.org/10.21203/rs.3.rs-47525/v1

License: (c) (i) This work is licensed under a Creative Commons Attribution 4.0 International License. Read Full License 


\section{Abstract}

Objectives: $\mathrm{Ca}^{2+}$ is critical for normal oocyte activation and fertilization, and any alteration to the $\mathrm{Ca}^{2+}$ homeostasis may lead to failed fertilization or even cell death. It has been shown that intracellular $\mathrm{Ca}^{2+}$ is increased in bovine and human oocytes when cultured in vitro. Additionally, ATP sensitive potassium channels have been characterised recently in human and Xenopus oocytes Glibenclamide a $\mathrm{K}_{\text {ATP }}$ channel blocker was shown to protect human oocytes from $\mathrm{Ca}^{+2}$ overloading. None of these studies have been conducted in mouse oocytes to determine if they are a suitable alternative to human oocytes in the research setting. Thus, this research note aims to demonstrate if cryopreserved metaphase II (MII) mouse oocytes show similar $\mathrm{Ca}^{+2}$ and plasmalemmal membrane potential dynamics to those in human oocytes. Also, to show if glibenclamide influences $\mathrm{Ca}^{+2}$, and plasmalemmal membrane potential in cryopreserved metaphase II mouse oocytes.

Results: our data did not show an increase in intracellular $\mathrm{Ca}^{2+}$ in untreated cryopreserved mouse oocytes loaded with Fluo-3 AM dye. However, an increase in the plasmalemmal membrane potential was noticed (hyperpolarization). Glibenclamide has shown no significant effect on $\mathrm{Ca}+2$, mitochondrial and plasmalemmal membrane potential.

\section{Introduction}

Research to date has not yet determined a major causative factor responsible for oocyte impairment in vitro (1). However, many studies have suggested that oocyte quality may be compromised by stimulation regimes, environmental and biological factors among others $(1,2)$. Existing research recognises that oocyte ageing and oxidative stress are the major causes of in vitro oocyte impairment (1). Several lines of evidence suggested that oxidative stress can be linked to $\mathrm{Ca}^{2+}$ homeostasis impairment, apoptosis, mitochondrial dysfunction, increased failed fertilization, and poor embryo development $(1,3-12) . \mathrm{Ca}^{2+}$ signals influence most of the principal events related to fertilization and embryo development in all investigated species (13). $\mathrm{Ca}^{2+}$ is a ubiquitous second messenger that synchronizes many cell functions such as gene expression and fertilisation (13). Fertilization is one of the most critical events in the oocytes in different maturation stages (14). Recently Fernandes et al. have examined the effects of in vitro stress on $\mathrm{Ca}^{2+}$ homeostasis in human oocytes, and they found that intracellular $\mathrm{Ca}^{2+}$ was increased in human oocytes when exposed to in vitro conditions (2). To prevent this increase in intracellular $\mathrm{Ca}^{2+}$, drugs targeting the ATP sensitive potassium channels $\left(\mathrm{K}_{\text {ATP }}\right.$ ) were applied to oocytes to investigate their cytoprotecting effects. Glibenclamide, a $\mathrm{K}_{\text {ATP }}$ channel blocker has been shown to reduce and prevent intracellular $\mathrm{Ca}^{2+}$ loading and thus provide cytoprotection in human oocytes (2). However, no studies have tested $\mathrm{Ca}^{2+}$ and plasmalemmal membrane potential dynamics in mouse oocytes. Therefore, it is important to study intracellular $\mathrm{Ca}^{2+}$ and $\mathrm{K}_{\text {ATP }}$ channels dynamics to further explain mouse oocyte ageing and sensitivity to in vitro oxidative stress. This study aims to determine $\mathrm{Ca}^{2+}$ and plasmalemmal membrane potential dynamics in cryopreserved mouse oocytes in vitro. The effect of glibenclamide on 
$\mathrm{Ca}^{2+}$ and membranes potential dynamics in cryopreserved mouse oocytes will also be investigated. Results of this study represent a single observation and they do not belong to a currently running project.

\section{Materials And Methods}

\section{MII mouse oocytes}

A total number of 56 cryopreserved MII mouse oocyte were used as the subjects of this study. Cryopreserved oocytes were purchased from Embryotech, US and no experiments were performed on animals. Therefore, no ethical approval was required to work on the cryopreserved mouse oocytes. All experiments performed at the research laboratory of the Clinical Embryology Department at the Medical School, Ninewells and took place from April 2018 to July 2018. At arrival, oocytes were frozen by utilizing a slow freeze method by the supplier (Embryotech, US). According to the supplier, purchased oocytes were harvested from superovulated female B6C3F-1 x B6D2F-1 mice at a regular predetermined hour, post-hCG injection. Before each experiment, oocytes were thawed at the research laboratory of the Clinical Embryology Department at the Medical School, Ninewells according to the supplier instructions. Briefly, straws containing 5-10 oocytes were removed from the cane and held for 1 minute in a $37^{\circ} \mathrm{C}$ waterbath and then removed and wiped dry. The contents of the straw were immediately expelled into a holding dish containing $1 \mathrm{ml}$ of HEPES buffered medium (Origio, Denmark). Finally, after the warming process, oocytes in the holding dish were incubated in a non-gassed incubator at $37^{\circ} \mathrm{C}$ until use. Only Metaphase II oocytes with normal morphology represented by presence of zuna pellucida, normal cytoplasm, and single polar body were included in this study. Oocytes with abnormal morpholoy and different maturation stages were excluded from the study subjects.

Oocytes were arbitrarily divided between experimental groups that were studied independently from each other in order to assess $\mathrm{Ca}^{2+}$ and plasmalemmal membrane potential levels:

i. Negative control (in the absence of any compound)

ii. Dimethyl sulfoxide DMSO 0.1\% (used to dissolve glibenclamide and FCCP)

iii. Glibenclamide (100 $\mu \mathrm{M}$ in $0.1 \%$ DMSO, a $\mathrm{K}_{\text {ATP }}$ channel blocker)

iv. Carbonyl cyanide-p-trifluoromethoxyphenylhydrazone (FCCP) $50 \mu \mathrm{M}$ in 0.1\% DMSO (oxidative phosphorylation inhibitor and chemical hypoxia inducer)

v. FCCP $50 \mu \mathrm{M}+$ Glibenclamide $100 \mu \mathrm{M}$ (this group was only included for oocytes stained with Fluo-3 AM )

\section{Laser Confocal Microscopy Imaging}

Oocytes were loaded with Fluo-3 AM Calcium indicator $(0.5 \mu \mathrm{g})$, and Di-8-ANEPPS plasmalemmal membrane potential probe $(10 \mu \mathrm{M})$. Stains were freshly prepared on the day of the experiments. After staining, oocytes were loaded into the wells of the ibidi slide (ibidi, Germany) containing $180 \mu \mathrm{l}$ of HEPES buffered media without Human Serum Albumin (to prevent oocyte from floating and moving during the 
analysis). No more than 5 oocytes were loaded into the slide well. After oocyte loading, the slide was transferred to the confocal laser microscope room by a transport incubator at $37^{\circ} \mathrm{C}$. The ibidi slide containing the oocytes was placed on the confocal laser microscope stage inside an environmental chamber. The temperature of the microscope chamber was maintained at $37^{\circ} \mathrm{C}$ during all the preparation and analysis times. Each oocyte imaged using laser confocal microscopy coupled to an inverted microscope (Leica TCS SP5 II, Milton Keynes, UK) with a $\times 10$ (numerical aperture 1.3) oil-immersion objective lens. The intensity of fluorescence of whole oocytes on the equatorial plane was measured. The microscope was calibrated by the green calibration slide before each experiment. The intensity of fluorescence was described in arbitrary units (AU) covering a range from 0 to $60000 \mathrm{AU}$. $\mathrm{Ca}^{2+}$ levels, plasmalemmal membrane potential and cell morphology were imaged every $10 \mathrm{~min}$ for $2 \mathrm{~h}$ using an Argon/UV laser (excitation 480-505 nm/emission 520-610 nm). Images were analysed using Leica Application Suite AF Lite software (Leica). The parameters of image acquisition were similar for all examined oocytes. Unless otherwise specified, all reagents and chemicals used in this study were purchased from Sigma-Aldrich.

\section{Statistical Analysis}

The normality and assumptions were calculated by using SigmaPlot version 4, from Systat Software, Inc., San Jose California USA to ensure the data were normally distributed. The Shapiro-Wilk statistical test was used for the normality testing.Repeated measures two-way Analysis of Variance (RM Two-way ANOVA) was performed as the data had two variables (time and intensity). This was carried out using GraphPad Prism version 7.00 for Windows, GraphPad Software, La Jolla California USA. Additionally, Tukey's multiple comparisons test was used to detect any statistical significance between the individual groups and the different time points within each group. A P value less than 0.05 was considered statistically significant.

\section{Results}

\section{Intracellular $\mathrm{Ca}^{+2}$ Changes in Cryopreserved MII Mouse Oocytes}

A total number of 32 cryopreserved mouse oocytes were loaded with Fluo-3 AM dye to monitor the changes in intracellular $\mathrm{Ca}^{+2}$ over time. A baseline measurement was taken for all the oocytes before the different treatments. After the baseline measurement, the oocytes were divided among different treatment groups.

In Figure 1A the untreated oocytes (negative controls) $(n=7)$ demonstrated no increase in intracellular $\mathrm{Ca}^{+2}$ over 120 minutes. The slight decrease in the fluorescent intensity ( $34.3 \pm 6 \mathrm{AU}$ to $21.6 \pm 5.4 \mathrm{AU}$ ) over time in the untreated group was statistically insignificant compared to the baseline measurement ( $\mathrm{P}$ value $=0.784)$ see Figure $1 \mathrm{~A}$. Also, there was no difference between negative control and oocytes treated with glibenclamide $(n=10)(P$ value= 0.996) (Figure 1A). This indicates that glibenclamide has no effect on $\mathrm{Ca}^{+2}$ in cryopreserved mouse oocytes see Figure 1A. FCCP $(n=6)$ and FCCP+Glibenclamide $(n=4)$ 
groups showed an increase in Fluo-3 AM intensity and that increase was statistically significant when compared to the untreated oocytes with $P$ values of 0.0004 and 0.0001 respectively (Figure 1A). No statistical difference was recorded between DMSO group $(n=5)$ and untreated oocytes (P value $=0.679)$ (Figure 1A). Figure 1B represents images of confocal laser microscopy for the oocytes of the different groups from depicted time points (magnification $x 10$ ). The variation in sample size amongst the groups was not intended for a specific reason but depended on oocyte availability at the experiment time.

\section{Plasmalemmal Membrane Potential Changes in Cryopreserved Mouse Oocytes}

To record plasmalemmal membrane potential changes, 24 cryopreserved oocytes were loaded with Di-8Anneps dye. A baseline measurement was also taken for all the oocytes before the different treatments. After the baseline measurement, the oocytes were divided among different treatment groups.

Figure 2A illustrates a spontaneous decrease in fluorescent intensity over 120 minutes in the negative control $(n=4)(23.3 \pm 0.7 \mathrm{AU}$ to $9.1 \pm 0.4 \mathrm{AU})$ with a P-value of $<0.0001$. That decrease in intensity indicates that plasmalemmal membranes of MII mouse oocytes experience significant hyperpolarisation (low membrane potential). The glibenclamide group $(n=10)$ also showed a significant decrease in fluorescent intensity over 120 minutes and when compared to the untreated group no significant difference was detected ( $P$ value= 0.782$)$ see Figure 2A. DMSO group $(n=5)$ showed similar intensity trends compared to the untreated oocytes and the glibenclamide group (figure 2A). Moreover, the FCCP group $(n=5)$ showed increased Di-8-Anneps intensity and that increase was statistically significant when compared with the untreated group $(P$-value $=0.002)$ see Figure $2 A$. Figure $2 B$ represents images of confocal laser microscopy for the oocytes of the different groups from depicted time points (magnification $\times 10$ ). The variation in sample size amongst the groups was not intended for a specific reason but depended on oocyte availability at the experiment time.

\section{Discussion}

In this study, we assessed the intracellular $\mathrm{Ca}^{2+}$ and plasmalemmal membrane potential trends over time in cryopreserved MII mouse oocytes to see if they show similar changes as those in human oocytes following data from Fernandes et al. study (2). Additionally, we investigated the glibenclamide effect on intracellular $\mathrm{Ca}^{2+}$, and plasmalemmal membranes in cryopreserved MII mouse oocytes compared to controls.

This study shows that there is no spontaneous increase in intracellular $\mathrm{Ca}^{2+}$ in untreated cryopreserved mouse oocytes loaded with Fluo-3 AM dye. Our results are not in agreement with Fernandes et al., findings (2). In Fernandes et al. study, they showed that human oocyte experience intracellular $\mathrm{Ca}^{2+}$ overloading in vitro (2). To the best of our knowledge, no previous study investigated $\mathrm{Ca}^{2+}$ dynamics in mouse oocyte before fertilization in vitro. Therefore, mouse oocytes may express different $\mathrm{Ca}^{2+}$ dynamics than those in human oocyte and are capable of sustaining $\mathrm{Ca}^{2+}$ levels in vitro. We also demonstrate that glibenclamide in the concentration of $100 \mu \mathrm{M}$ does not affect the intracellular $\mathrm{Ca}^{2+}$ trend in mouse 
oocytes. Our results are in agreement with $\mathrm{Li}$ et al. study, which showed that glibenclamide did not affect the resting $\mathrm{Ca}^{2+}$ of Raw 264.7 macrophages (15). This observation could be explained as glibenclamide might specifically target mitochondrial $\mathrm{K}_{\text {ATP }}$ channels, not the plasmalemmal ones. Therefore, future research should consider both mitochondrial and plasmalemmal $\mathrm{K}_{\text {ATP }}$ channels with $\mathrm{Ca}^{2+}$ regulation in oocytes. Our data also demonstrates that plasmalemmal membrane potential significantly declined (therefore hyperpolarized) in all the groups, except for the positive control (FCCP) group. This observation could not be explained concerning oocytes as to the best of our knowledge no previous published studies investigated plasmalemmal membrane potential in oocytes. Glibenclamide did not cause any changes in the hyperpolarized plasmalemmal membrane potential compared to the control groups. This observation suggests that glibenclamide might function exclusively on mitochondrial $\mathrm{K}_{\text {ATP }}$ channels, as suggested by Fernandes et al. study (2). To confirm this, higher concentrations of glibenclamide and longer incubation time are needed.

\section{Conclusions}

Our data show no spontaneous $\mathrm{Ca}^{2+}$ increase in untreated cryopreserved mouse oocytes loaded with Fluo-3 AM dye in vitro. We also demonstrate that glibenclamide has no effect on $\mathrm{Ca}^{2+}$ homeostasis in cryopreserved mouse oocytes. Interestingly, this study shows that cryopreserved mouse oocytes express plasmalemmal $\mathrm{K}_{\text {ATP }}$ channel hyperpolarization. This study also suggests the presence of $\mathrm{K}_{\text {ATP }}$ channels in the plasma membrane of mouse oocytes as no previous research showed their expression in mouse oocytes before. Finally, we show that $\mathrm{Ca}^{2+}$ dynamics in mouse oocytes are not similar to those in human oocytes. Therefore, cryopreserved mouse oocyte cannot represent human oocytes as a model to investigate $\mathrm{Ca}^{2+}$ dynamics in vitro.

\section{Limitations}

- Unavailability of fresh mouse oocytes to compare with the cryopreserved ones.

- The low number of oocytes tested represents a limitation in the analysis and interpretation of the study findings.

- $\mathrm{Ca}^{2+}$ dynamics tested directly after thawing and staining of cryopreserved oocytes, therefore, longer incubation times before staining might give more insight into $\mathrm{Ca}^{2+}$ dynamics in Mll cryopreserved oocytes.

- Only one concentration of glibenclamide was tested (100 $\mu \mathrm{M}$ in $0.1 \%$ DMSO) due to the low sample size.

\section{Abbreviations}

AU: arbitrary, $\mathrm{Ca}^{+2}$ : calcium, DMSO: Dimethyl sulfoxide, $\mathrm{K}_{\text {ATP: }}$ ATP sensitive potassium channels, MII: Metaphase II, mtDNA: mitochondrial DNA, HAS: Human Serum Albumin, HEPES: (4-(2-hydroxyethyl)-1- 
piperazineethanesulfonic acid) FCCP: carbonyl cyanide 4 (trifluoromethoxy)phenylhydrazone, DMSO: Dimethyl sulfoxide, SEM: stander error of the mean.

\section{Declarations}

Ethics approval and consent to participate

Not applicable

Consent for publication

Not applicable

\section{Availability of data and materials}

The data analysed during the current study are available from the corresponding author on reasonable request.

\section{Competing interests}

The authors declare that they have no competing interests

\section{Funding}

Not applicable

\section{Authors' contributions}

Conceptualization, OFA and TM; Methodology, OFA and TM; Investigation, OFA; Resources, TM; WritingOriginal Draft, OFA; Writing-Review \& Editing, OFA and TM; Visualization, OFA; Supervision, TM. All authors read and approved the final manuscript.

\section{Acknowledgements}

Not applicable

\section{References}

1. Lord T, Aitken RJ. Oxidative stress and ageing of the post-ovulatory oocyte. Reproduction. 2013 Oct 21;146(6):R217-27.

2. Fernandes G, Dasai N, Kozlova N, Mojadadi A, Gall M, Drew E, et al. A spontaneous increase in intracellular $\mathrm{Ca}^{2+}$ in metaphase II human oocytes in vitro can be prevented by drugs targeting ATPsensitive $K^{+}$channels. Hum Reprod. 2015 Dec;18(2):dev300. 31. 
3. Brisson M, Foster C, Wipf P, Joo B, Tomko RJ, Nguyen T, et al. Independent Mechanistic Inhibition of Cdc25 Phosphatases by a Natural Product Caulibugulone. Mol Pharmacol. 2006 Oct 11;71(1):18492.

4. Petrosillo G, Ruggiero FM, Pistolese M, Paradies G. Ca ${ }^{2}$ +-induced Reactive Oxygen Species Production Promotes Cytochrome $c$ Release from Rat Liver Mitochondria via Mitochondrial Permeability Transition (MPT)-dependent and MPT-independent Mechanisms. J Biol Chem. 2004 Dec 17;279(51):53103-8.

5. Batchelor TT, Sorensen AG, di Tomaso E, Zhang W-T, Duda DG, Cohen KS, et al. AZD2171, a panVEGF receptor tyrosine kinase inhibitor, normalizes tumor vasculature and alleviates edema in glioblastoma patients. Cancer Cell. 2007;11(1):83-95.

6. Chan TM, Chen E, Tatoyan A, Shargill NS, Pleta M, Hochstein P. Stimulation of tyrosine-specific protein phosphorylation in the rat liver plasma membrane by oxygen radicals. Biochem Biophys Res Commun. 1986 Sep;14(2):439-45. 139(.

7. Chaube SK, Prasad PV, Thakur SC, Shrivastav TG. Hydrogen peroxide modulates meiotic cell cycle and induces morphological features characteristic of apoptosis in rat oocytes cultured in vitro. Apoptosis. 2005 Aug;10(4):863-74.

8. Haverfield J, Nakagawa S, Love D, Tsichlaki E, Nomikos M, Lai FA, et al. Ca2 + dynamics in oocytes from naturally-aged mice. Sci Rep. 2016 May;20(1):19357. 6(.

9. Kikuchi K, Naito K, Noguchi J, Kaneko H, Tojo H. Maturation/M-Phase Promoting Factor Regulates Aging of Porcine Oocytes Matured In Vitro. Cloning Stem Cells. 2002 Sep;4(3):211-22.

10. Lord T, Nixon B, Jones KT, Aitken RJ. Melatonin Prevents Postovulatory Oocyte Aging in the Mouse and Extends the Window for Optimal Fertilization In Vitro1. Biol Reprod. 2013 Mar 1;88(3):67.

11. Melov S, Coskun P, Patel M, Tuinstra R, Cottrell B, Jun AS, et al. Mitochondrial disease in superoxide dismutase 2 mutant mice. Proc Natl Acad Sci U S A. 1999 Feb 2;96(3):846-51.

12. Monteiro HP, Ivaschenko Y, Fischer R, Stern A. Inhibition of protein tyrosine phosphatase activity by diamide is reversed by epidermal growth factor in fibroblasts. FEBS Lett. 1991 Dec 16;295(13):146-8.

13. Miao Y-L, Williams CJ. Calcium signaling in mammalian egg activation and embryo development: the influence of subcellular localization. Mol Reprod Dev. 2012 Nov;79(11):742-56.

14. Wakai T, Vanderheyden V, Fissore RA. Ca2 + signaling during mammalian fertilization: requirements, players, and adaptations. Cold Spring Harb Perspect Biol. 2011 Apr 1;3(4):a006767.

15. Li D, Ma Z, Fu Z, Ling M, Yan C, Zhang Y. Glibenclamide decreases ATP-induced intracellular calcium transient elevation via inhibiting reactive oxygen species and mitochondrial activity in macrophages. PLoS One. 2014;9(2):e89083.

\section{Figures}



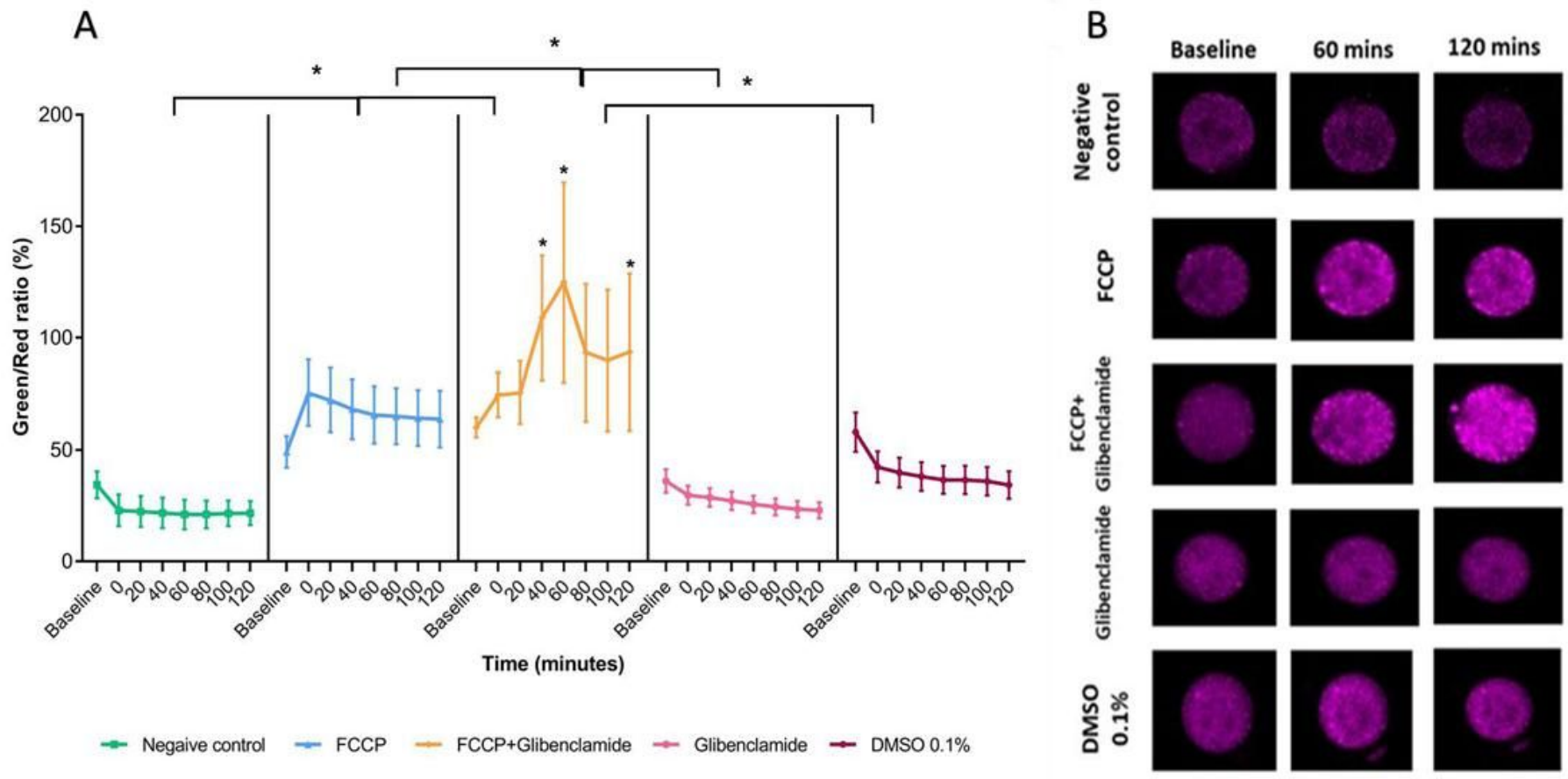

\section{Figure 1}

Ca2+ changes over time in MII cryopreserved mouse oocytes. (A) Fluo-3 AM means intensity changes over time (minutes) in cryopreserved mouse oocytes. Negative control $n=7$ oocytes, FCCP $n=6$ oocytes, FCCP+Glibenclamide $n=4$ oocytes, Glibenclamide $n=10$ oocytes and DMSO $n=5$ oocytes. RM Two-way ANOVA and Tukey's Multiple comparison tests used to determine statistical significance between the individual groups and to compare each time point within the single group to the baseline point. ${ }^{*}=\mathrm{P}$ value $>0.05$. Error bars represent SEM. (B) Images of confocal laser microscopy for the oocytes of the different groups from depicted time points (magnification $x 10$ ). 


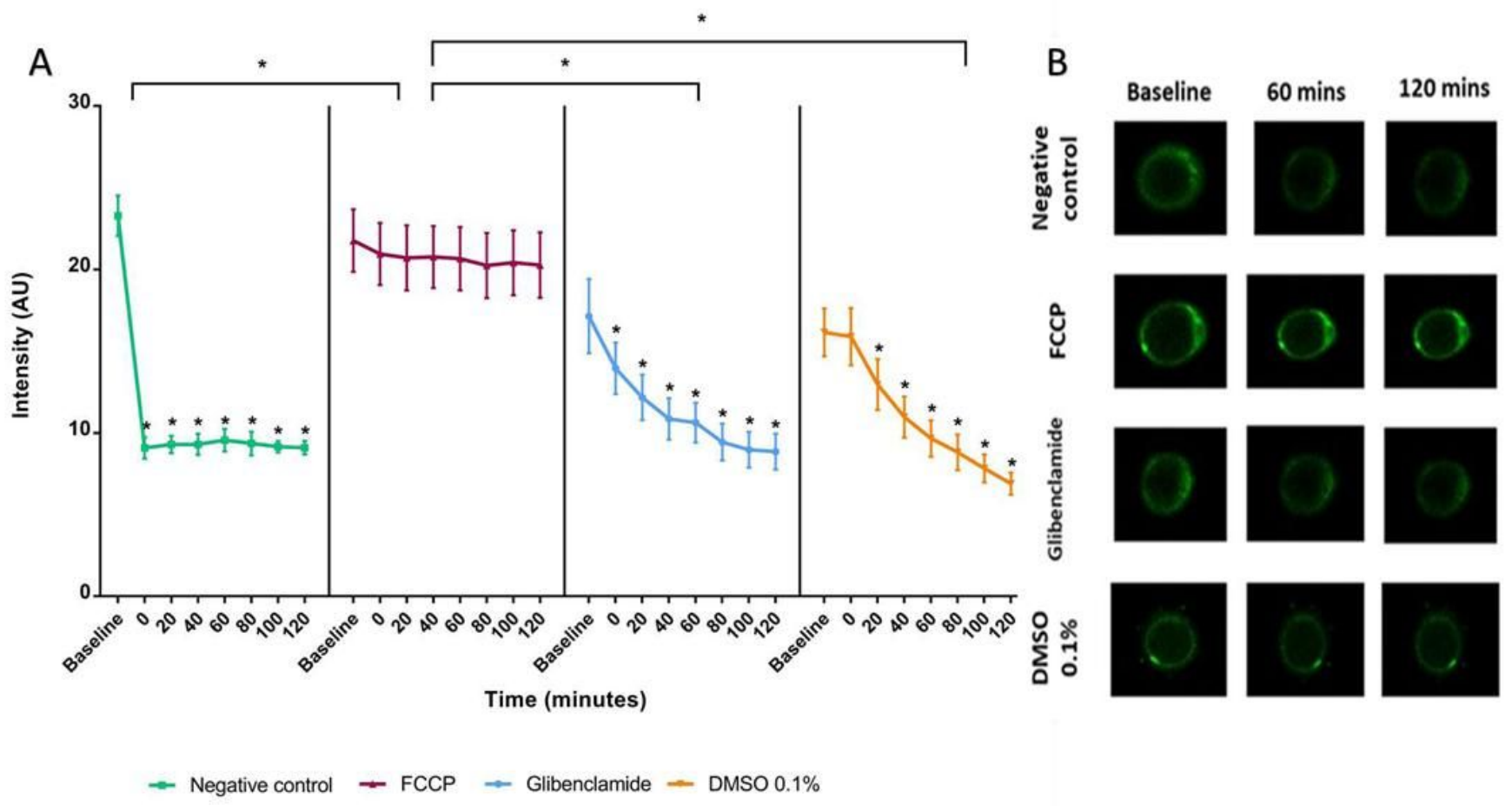

Figure 2

Di-8-Anneps changes over time in Mll cryopreserved mouse oocytes. (A) Di-8-Anneps mean intensity changes over time (minutes) in cryopreserved mouse oocytes. Negative control $n=4$ separate oocytes, FCCP $n=5$ separate oocytes, Glibenclamide $n=10$ separate oocytes and DMSO $n=5$ separate oocytes. RM Two-way ANOVA and Tukey's Multiple comparison tests used to determine statistical significant between the individual groups and to compare each time point within the single group to the baseline point. * $=P$ value $>0.05$. Error bars represent SEM. (B) Images of confocal laser microscopy for the oocytes of the different groups from depicted time points (magnification $x 10$ ).

\section{Supplementary Files}

This is a list of supplementary files associated with this preprint. Click to download.

- AuthorChecklistFull.pdf 